\title{
Mini Nutritional Assessment Scale-Short Form can be useful for frailty screening in older adults
}

This article was published in the following Dove Medical Press journal:

Clinical Interventions in Aging

\author{
Pinar Soysal' \\ Nicola Veronese ${ }^{2,3}$ \\ Ferhat Arik ${ }^{4}$ \\ Ugur Kalan ${ }^{4}$ \\ Lee Smith ${ }^{5}$ \\ Ahmet Turan Isik ${ }^{6}$ \\ 'Department of Geriatric Medicine, \\ Faculty of Medicine, Bezmialem Vakif \\ University, Istanbul, Turkey; ${ }^{2}$ National \\ Research Council, Neuroscience \\ Institute, Aging Branch, Padova, Italy; \\ ${ }^{3}$ Department of Geriatric Care, \\ Ortho Geriatrics and Rehabilitation, \\ E.O. Galliera Hospital, National \\ Relevance and High Specialization \\ Hospital, Genova, Italy; ${ }^{4}$ Department \\ of Internal Medicine, Geriatric \\ Center, Kayseri Education and \\ Research Hospital, Kayseri, Turkey; \\ ${ }^{5}$ The Cambridge Centre for Sport \\ and Exercise Sciences, Anglia Ruskin \\ University, Cambridge, UK; ${ }^{6}$ Unit \\ for Aging Brain and Dementia, \\ Department of Geriatric Medicine, \\ Faculty of Medicine, Dokuz Eylul \\ University, Izmir, Turkey
}

\begin{abstract}
Aim: Mini Nutritional Assessment-Short Form (MNA-SF) is used to assess nutritional status in older adults, but it is not known whether it can be used to define frailty. This study was aimed to investigate whether or not MNA-SF can identify frailty status as defined by Fried's criteria. Methods: A total of 1,003 outpatients (aged 65 years or older) were included in the study. All patients underwent comprehensive geriatric assessment. Frailty status was evaluated by Fried's criteria: unintentional weight loss, exhaustion, low levels of activity, weakness, and slowness. One point is assigned for each criterion, and frailty status is identified based on the number of points scored: 0 points, not frail; $1-2$ points, pre-frail; $\geq 3$ points, frail. A total score of MNA-SF $<8,8-11$, and $>11$ indicates malnutrition, risk of malnutrition, and no malnutrition, respectively.

Results: Of the 1,003 outpatients (mean age $74.2 \pm 8.5$ years), 313 participants $(31.2 \%)$ were considered frail and 382 (38.1\%) pre-frail. Among frail and pre-frail patients, $49.2 \%$ and $25.1 \%$ were at risk of malnutrition and $22.0 \%$ and $1.6 \%$ were malnourished, respectively. MNA-SF with a cut-off point of 11.0 had a sensitivity of $71.2 \%$ and a specificity of $92.8 \%$ for the detection of frail participants, and with a cut-off point of 13 had a sensitivity of $45.7 \%$ and a specificity of $78.3 \%$ for the detection of pre-frailty. The area under the curve for MNA-SF was estimated to be 0.906 and 0.687 for frailty and pre-frailty, respectively.
\end{abstract}

Conclusion: MNA-SF can be useful for frailty screening in older adults.

Keywords: malnutrition, frailty, nutritional status, pre-frailty

\section{Introduction}

Malnutrition and frailty are two important geriatric syndromes, and in recent years, it has been speculated that these two syndromes are likely to be associated. ${ }^{1,2}$ There are several plausible mechanisms that may explain such an association. Weight loss can aid in the development of both malnutrition and frailty. An imbalance between energy intake and expenditure can cause muscle weakness, which may be associated with poor muscle strength, slowness, exhaustion, and reduced functional activities. . $^{3,4}$ Additionally, higher protein and specific micronutrient intake, and dietary pattern with more phytonutrient-rich plant foods, omega-3-rich fish, and other protein-rich foods are associated with lower incidence of frailty. ${ }^{5,6}$ Moreover, malnutrition-related negative outcomes such as sarcopenia, depression, cognitive impairment, falls, delayed immune response, and increased risk of infection further aid in the development of frailty. ${ }^{7-11}$ When older patients develop frailty, their muscle protein catabolism increases and age-related loss of muscle mass enhances, which results in impaired mobility and dependency on others. ${ }^{12}$ Therefore, frail patients have more difficulties in the selection, preparation, and intake of food, and they have a greater loss of appetite, eating problems, and swallowing problems., ${ }^{3,13}$ As a result, a vicious cycle develops
Correspondence: Pinar Soysal Department of Geriatric Medicine, Faculty of Medicine, Bezmialem Vakif University, Adnan Menderes Bulvarı (Vatan Street) Fatih 34093, Istanbul, Turkey

Tel +902125232288

Fax +902124531870

Email dr.pinarsoysal@hotmail.com 
between malnutrition and frailty. The screening of frailty and nutritional status as well as early diagnosis is needed to implement appropriate interventions.

The Mini-Nutritional Assessment Scale-Short Form (MNA-SF) $)^{14}$ is a screening scale used to assess nutritional status, but it also includes queries regarding other geriatric issues, including cognitive impairment and depression, mobility, acute disease or psychological stress, weight loss, and food intake, which can define not only malnutrition but can also provide information regarding frailty and pre-frailty. ${ }^{14}$ Moreover, analysis by MNA-SF is much quicker and easier when compared to measurement by Fried's phenotypic criteria. Also, it provides additional information about some of the health issues, such as cognition, which are not evaluated by Fried's criteria. ${ }^{15}$ Additionally, there are only a few studies that have found an association between MNA-SF scores and frailty. ${ }^{16,17}$

Therefore, this study was aimed to investigate whether or not the MNA-SF can identify frailty status as defined by Fried's criteria. ${ }^{18}$

\section{Methods}

\section{Participants}

A total of 1,402 outpatients who were admitted to a geriatric outpatient clinic for any health issue between February 2017 and June 2018 were included in the study. Information regarding patients' age, sex, education level, comorbidities, and number of drugs used were recorded. All the patients underwent comprehensive geriatric assessment (CGA), including Mini-Mental State Examination (MMSE), ${ }^{19}$ Geriatric Depression Scale (GDS) ${ }^{20}$ for neurocognitive evaluation, basic and instrumental activities of daily living (BADL and IADL, respectively) for functionality evaluation, ${ }^{21}$ Tinetti Performance-Oriented Mobility Assessment (POMA) for mobility evaluation, ${ }^{22}$ and MNA-SF for nutritional evaluation. ${ }^{23}$

Patients who did not complete CGA; patients who refused to participate; patients who had a history of severe illness that may impair general health status, such as acute renal failure, acute liver failure, acute cerebrovascular event, and sepsis; and patients under 65 years of age were excluded. Dementia was diagnosed according to Diagnostic and Statistical Manual of Mental Disorders, Fifth Edition, criteria and patients with severe dementia, who were classified as Clinical Dementia Rating 3, were also excluded as these individuals might not be able to accurately recall responses for questions mentioned in Fried and MNA-SF questionnaires. Moreover, the reliability of hand grip strength is low for older patients with dementia. ${ }^{24,25}$ After CGA was performed by a geriatrician, 1,003 patients who did not meet the exclusion criteria were included in this study. Informed consent was provided by each participant or a legal guardian before participating in the study.

\section{MNA-SF and diagnosis of frailty}

The MNA-SF test comprises simple measurements and six questions that can be completed in less than 5 minutes: anthropometric measurements (body mass index, weight loss); global assessment (mobility); and dietary questionnaire and subjective assessment (food intake, neuropsychological problems, acute disease). A total score of MNA-SF $<8,8-11$, and $>11$ indicates malnutrition, risk of malnutrition, and no malnutrition, respectively. ${ }^{23,26}$

Fried et al in 2001 defined the frailty status based on five dimensions of frailty phenotype. These criteria include: shrinking, exhaustion, low levels of physical activity, weakness, and slowness. One point is assigned for each criterion: 0 points, not frail/robust; $1-2$ points, pre-frail; $\geq 3$ points, frail. ${ }^{18}$ Weakness, assessed by grip strength of the dominant hand with a dynamometer, was defined as less than or equal to the cut-off points according to the sex and BMI points determined by Fried et al. Shrinking was defined as unintentional weight loss of $4.5 \mathrm{~kg}$ or $>5 \%$ of baseline body weight within the previous year. Patients were considered to have low physical activity if they had performed no physical activity, or rarely had short walks, or spent most of the time sitting in the previous year. ${ }^{18,27}$ Gait speed was evaluated by using $4 \mathrm{~m}$ walking test, and it was regarded as slow if the time recorded was less than or equal to the cut-off points according to sex and height. The exhaustion criterion was met if the answer was, "Much or most of the time" when asked, "How often in the last week did you feel this way" to either of the following two statements: "I felt that everything I did was an effort" and "I could not get going." 18

The investigation conformed to the Declaration of Helsinki. Written informed consent was received from the elderly subjects themselves or their proxy, and ethical approval was given by Erciyes University Institutional Board (2017/405).

\section{Statistical analyses}

SPSS statistics (version 17.0) was used to perform the analysis. Nominal variables were assessed by Pearson's chisquared test. Continuous variables with normal distribution were analyzed using one-way ANOVA followed by a post hoc test, and the Kruskal-Wallis test was used to assess the 
presence of non-normal distribution. Variances in more than two groups were assessed by post hoc Tukey's test. The area under the receiver operating characteristic (ROC) curve was used to test the predictive accuracy to determine a suitable cut-off point. The test was repeated two times at an interval of 3 weeks by another researcher. Internal consistency was analyzed using Cronbach's alpha test. Cut-off scores were assessed by constructing the ROC curve. All analyses were conducted for both frail and pre-frail groups separately. Sensitivity, specificity, and positive (PPVs) and negative predictive values (NPVs) were calculated for different cut-off scores of MNA-SF to identify pre-frail and frail older adults. For $P<0.05$, results were considered statistically significant.

\section{Results}

Of the 1,003 outpatients (mean age $74.2 \pm 8.5$ years), $73.5 \%$ were female.

A total of 313 participants (31.2\%) were considered frail, $382(38.1 \%)$ pre-frail, and $308(30.7 \%)$ non-frail. About $6.8 \%$ of the patients were found to be malnourished, $33.0 \%$ were at risk for malnutrition, and $60.2 \%$ had a normal nutritional status. BADL, IADL, MMSE, POMA, and MNA-SF scores were lower, and the GDS and timed up and go test scores were higher in the frail group than in both pre-frail and robust groups (for each group, $P<0.05$ ). Descriptive characteristics of the participants are given in Table 1.

All the MNA-SF items that indicate malnutrition and total scores were significantly higher in frailty and pre-frailty groups than in the robust group $(P<0.05)$.

Among frail and pre-frail patients, $49.2 \%$ and $25.1 \%$ were at risk of malnutrition and $22.0 \%$ and $1.6 \%$ were malnourished, respectively $(P<0.05)$ (Table 2$)$.

The Cronbach's alpha for the internal consistency of the MNA-SF was 0.730 , and the interclass correlation coefficient for the test-retest reliability was 0.776 . MNA-SF with a cut-off point of 11.0 showed a sensitivity of $71.2 \%$ and a specificity of $92.8 \%$ for the detection of frail participants, and a cut-off point of 13 showed a sensitivity of $45.7 \%$ and a specificity of $78.3 \%$ for the detection of pre-frailty. The sensitivity, specificity, PPV, and NPV of the MNA-SF according to the Fried's criteria are shown in Table 3.

The area under the ROC curve for MNA-SF was estimated to be 0.906 and 0.687 for frailty and pre-frailty, respectively (Figure 1).

\section{Discussion}

The present study found that frailty status is associated with the nutritional status, and that MNA-SF shows strong
Table I Descriptive characteristics of the participants

\begin{tabular}{|c|c|}
\hline Age (years) (mean \pm SD) & $74.2 \pm 8.5$ \\
\hline Gender, \% (female/male) & $73.5 / 26.5$ \\
\hline Education (years) & $1.1 \pm 1.3$ \\
\hline Body mass index $\left(\mathrm{kg} / \mathrm{m}^{2}\right)(\operatorname{mean} \pm \mathrm{SD})$ & $33.1 \pm 42.0$ \\
\hline \multicolumn{2}{|l|}{ Comorbidities (\%) } \\
\hline - Hypertension & 65.5 \\
\hline - Diabetes mellitus & 35.2 \\
\hline - Congestive heart failure & 6.5 \\
\hline - Coronary artery disease & 13.6 \\
\hline - COPD & 17.6 \\
\hline - Osteoarthritis & 18.0 \\
\hline - Dementia & 7.1 \\
\hline - Dysphagia & 5.2 \\
\hline - Depression & 24.4 \\
\hline \multicolumn{2}{|l|}{ Geriatric assessment (mean $\pm S D)$} \\
\hline - MMSE & $24.3 \pm 4.6$ \\
\hline - BADL & $89.5 \pm 33.1$ \\
\hline - IADL & $17.3 \pm 7.4$ \\
\hline - GDS & $4.8 \pm 4.2$ \\
\hline - POMA & $27.4 \pm 8.6$ \\
\hline - TUG (seconds) & $14.1 \pm 9.7$ \\
\hline
\end{tabular}

Abbreviations: BADL, basic activity of daily living (0 [worst]-100 [best]); GDS, Geriatric Depression Scale (I5 [worst]-0 [best]); IADL, instrumental activity of daily living (0 [worst]-23 [best]); MMSE, Mini-Mental State Examination ( 0 [worst]-30 [best]); POMA, Performance-Oriented Mobility Assessment (0 [worst]-28 [best]); TUG, timed up and go test; SD, standard deviation.

correlation with Fried's frailty criteria in older adults. Therefore, MNA-SF can be useful in identifying frail and pre-frail patients against the Fried's frailty phenotype as a reference standard.

Frailty is a common geriatric syndrome. In our study, the prevalence of frail and pre-frail patients was found to be $31.6 \%$ and $36.2 \%$, respectively. The high prevalence of frailty might be related to low educational level and low socioeconomic status of the Turkish community's older patients, which can be attributed to poor dietary intake/ compliance, and due to the fact that the study population mainly consisted of older adults who had been admitted to the geriatric center for some health issue, and hence do not represent the whole community. ${ }^{28-30}$

A number of scales have been validated to help in the identification of frail subjects, but there is no consensus regarding which frailty evaluation tool should be used for the assessment of frailty status. Many factors can lead to the development of frailty, but malnutrition plays an important role. ${ }^{3}$ Malnutrition is characterized by deficiency in nutrient absorption or a decrease in nutrient intake, which can cause 
Table 2 Relationship between items of MNA-SF and frailty status

\begin{tabular}{|c|c|c|c|c|}
\hline MNA-SF items & Robust (29.8\%) & Pre-frail (38.1\%) & Frail (32.1\%) & $P$-value \\
\hline \multicolumn{5}{|l|}{ Malnutrition parameters } \\
\hline MNA-SF scores & $13.4 \pm 1.0$ & $12.3 \pm 1.8$ & $9.6 \pm 2.7$ & $<0.001$ \\
\hline Risk of malnutrition (\%) & 7.1 & 25.1 & 49.2 & $<0.001$ \\
\hline Malnutrition (\%) & 0 & 1.6 & 22.0 & $<0.001$ \\
\hline \multicolumn{5}{|l|}{ MNA-SF parameters } \\
\hline \multicolumn{5}{|l|}{ Decrease in food intake (\%) } \\
\hline Severe decrease & 1.3 & 1.8 & 14.1 & $<0.001$ \\
\hline Moderate decrease & 9.1 & 16.2 & 42.8 & \\
\hline No decrease & 89.6 & 81.9 & 43.1 & \\
\hline \multicolumn{5}{|l|}{ Weight loss (\%) } \\
\hline Weight loss greater than $3 \mathrm{~kg}$ & 2.3 & 8.4 & 29.7 & $<0.001$ \\
\hline Does not know & 1.6 & 5.0 & 10.5 & \\
\hline Weight loss between I and $3 \mathrm{~kg}$ & 3.6 & 5.5 & 10.5 & \\
\hline No weight loss & 92.5 & 81.2 & 44.4 & \\
\hline \multicolumn{5}{|l|}{ Mobility (\%) } \\
\hline Bed or chair bound & 0 & 0 & 4.5 & $<0.001$ \\
\hline Able to get out of bed/chair but does not go out & 0.3 & 5.5 & 30.4 & \\
\hline Goes out & 99.7 & 94.5 & 65.2 & \\
\hline \multicolumn{5}{|l|}{ Suffered psychological stress or acute disease (\%) } \\
\hline Yes & 9.1 & 11.5 & 24.6 & $<0.001$ \\
\hline No & 90.9 & 88.5 & 75.4 & \\
\hline \multicolumn{5}{|l|}{ Neuropsychological problems (\%) } \\
\hline Severe dementia or depression & 3.9 & 30.1 & 49.2 & $<0.001$ \\
\hline Mild dementia & 2.9 & 11.5 & 18.8 & \\
\hline No psychological problems & 93.2 & 58.4 & 32.0 & \\
\hline \multicolumn{5}{|l|}{ Body mass index (BMI) (\%) } \\
\hline $\mathrm{BMI}<19$ & 0.3 & 0.3 & 3.5 & $<0.001$ \\
\hline BMI I9 to $<21$ & 0.7 & 2.1 & 7.0 & \\
\hline BMI 2 I to $<23$ & 0.3 & 2.9 & 7.0 & \\
\hline $\mathrm{BMI} \geq 23$ & \begin{tabular}{|l|}
98.7 \\
\end{tabular} & 94.7 & 82.5 & \\
\hline
\end{tabular}

Abbreviations: MNA, Mini Nutritional Assessment; MNA-SF, MNA-Short Form.

many negative outcomes and consequently lead to frailty, if this condition is not treated or treatment is delayed., ${ }^{3,89}$ Thus, nutritional screening is very important for definition of frailty and frail older adults represent primary targets for nutritional therapy. ${ }^{31}$ Both MNA and MNA-SF are validated and effective tools for malnutrition screening, and when developing MNA, Vellas et al, different from previous scales, the two can use screening nutritional status for the frail older patients. ${ }^{23}$
Furthermore, in a study by Lilimand, MNA-SF was found to be an effective and valid screening scale to define malnutrition in frail older adults. ${ }^{31}$

Until now, there have been few studies investigating associations between MNA-SF scores and frailty, and these studies have found significant associations between nutritional status and frailty status. ${ }^{3,24,32}$ One of them carried out by Chang et $\mathrm{al}^{17}$ showed that community-dwelling older

Table 3 The sensitivity, specificity, NPVs, and PPVs of MNA-SF

\begin{tabular}{|l|l|l|l|l|l|l|}
\hline & Cut-off & Diagnosis & Sensitivity (\%) (Interval) & Specificity (\%) (Interval) & PPV (\%) (Interval) & NPV (\%) (Interval) \\
\hline \multirow{2}{*}{ MNA-SF } & 13.0 & Pre-frail & $45.71(39.77-51.75)$ & $78.32(73.09-82.96)$ & $67.37(61.55-72.0)$ & $59.57(56.57-62.51)$ \\
\cline { 2 - 7 } & 11.0 & Frail & $71.25(65.89-76.20)$ & $92.86(89.39-95.47)$ & $91.02(87.07-93.85)$ & $76.06(72.69-79.14)$ \\
\hline
\end{tabular}

Abbreviations: MNA, Mini Nutritional Assessment; MNA-SF, MNA-Short Form; PPV, positive predictive value; NPV, negative predictive value. 
A

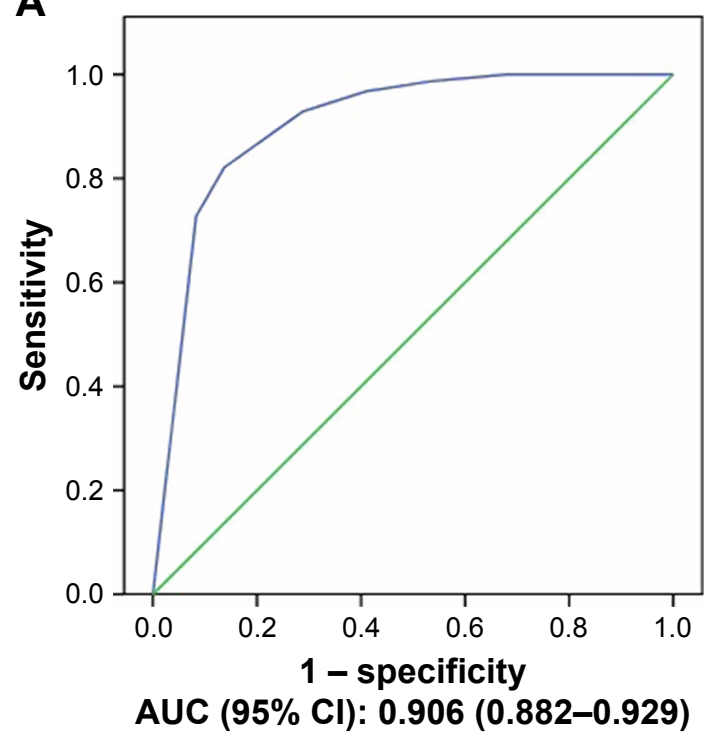

B

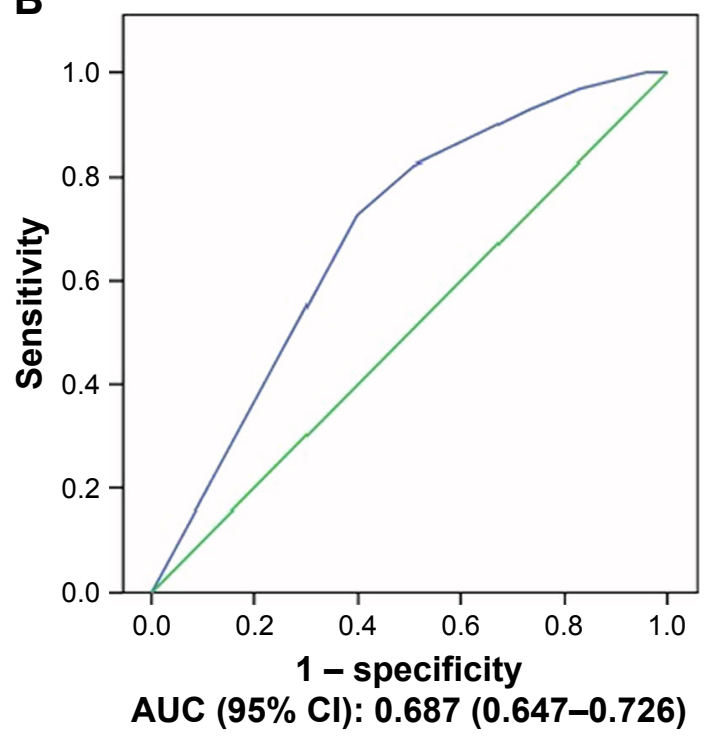

Figure I Receiver operating characteristic curve (ROC) analysis of the MNA-SF to detect (A) frailty and (B) pre-frailty. Abbreviations: AUC, area under the curve; MNA, Mini-Nutritional Assessment; MNA-SF, MNA-Short Form.

people who were frail had a high risk of malnutrition when evaluated by MNA-SF. Another study by Jürschik et al, including 640 community-dwelling elders, revealed that the higher the score of both MNA and MNA-SF, the higher was the frailty index score; thus, it was suggested that both tests could be useful to identify frail older adults. ${ }^{16}$ Similar to these studies, we also found that frailty and pre-frailty were strongly associated with nutritional status, as evaluated by MNA-SF. However, we showed that there was a strong relationship between each of the MNA-SF items and frailty status and that MNA-SF exhibited high specificity and high sensitivity in detecting not only malnutrition, but also frailty. This could be explained by several reasons. Firstly, even if MNA-SF is a screening tool for nutritional status, it also includes queries for the existence of dementia and depression, which are strongly associated with frailty.,33 Secondly, weight loss and decreased food intake, two items on the MNA-SF, can contribute to the development of both malnutrition and frailty, and an imbalance between energy intake and expenditure leads to muscle weakness, which can be correlated with all of the frailty criteria including exhaustion, poor muscle strength, decreased functional activities, and slowness. ${ }^{4}$ Thirdly, assessment of suffering from psychological stress or acute disease, another item of MNA-SF, may also be critical in the determination of frailty status owing to the fact that "mood and motivational frailty" is a part of physical and cognitive frailty, and frail patients usually tend to suffer from acute diseases and hospitalization. ${ }^{7,32,34}$ Lastly, frailty increases age-related loss of muscle mass and muscle protein catabolism resulting in impaired mobility, which is also assessed by MNA-SF. ${ }^{23,35}$ All these features of MNA-SF can explain why its specificity and sensitivity in detecting frailty and pre-frailty are high.

On the other hand, our study revealed that the best cut-off values for detection of frailty and pre-frailty were higher than those for detection of malnutrition and risk of malnutrition (11 vs 13 and 7 vs 11, respectively). Therefore, frailty and pre-frailty should be evaluated even if an older person has a good nutritional status.

The present study has some limitations. First of them is the cross-sectional design. Second is that only physical frailty was evaluated, and cognitive frailty was not evaluated. Third, patients with dementia were excluded, since the frailty phenotype has not been validated in subjects with major cognitive impairment. The strength of our study is the inclusion of a large sample size.

\section{Conclusion}

MNA-SF is a fast, simple, and sensitive method for screening both frailty and malnutrition. Therefore, MNA-SF can easily be used for the older adults by clinicians to determine frailty status as well as nutritional status. Thus, two geriatric syndromes, frailty and malnutrition, can be identified by MNA-SF simultaneously in geriatrics practice.

\section{Acknowledgments}

This research did not receive any specific grant from funding agencies of the public, commercial, or not-for-profit sectors. 


\section{Disclosure}

The authors report no conflicts of interest in this work.

\section{References}

1. Ates Bulut E, Soysal P, Isik AT. Frequency and coincidence of geriatric syndromes according to age groups: single-center experience in Turkey between 2013 and 2017. Clin Interv Aging. 2018;13:1899-1905.

2. Machon M, Mateo-Abad M, Vrotsou K, et al. Dietary patterns and their relationship with frailty in functionally independent older adults. Nutrients. 2018;10:4. doi:10.3390/nu10040406

3. Lorenzo-Lopez L, Maseda A, de Labra C, Regueiro-Folgueira L, Rodriguez-Villamil JL, Millan-Calenti JC. Nutritional determinants of frailty in older adults: a systematic review. BMC Geriatr. 2017;17(1): 108. doi:10.1186/s12877-017-0515-3

4. Abellan van Kan G, Rolland Y, Houles M, Gillette-Guyonnet S, Soto M, Vellas B. The assessment of frailty in older adults. Clin Geriatr Med. 2010;26(2):275-286. doi:10.1016/j.cger.2010.02.002

5. Shikany JM, Barrett-Connor E, Ensrud KE, et al. Macronutrients, diet quality, and frailty in older men. J Gerontol A Biol Sci Med Sci. 2014;69(6):695-701. doi:10.1093/gerona/glt196

6. Beasley JM, LaCroix AZ, Neuhouser ML, et al. Protein intake and incident frailty in the Women's Health Initiative observational study. $J$ Am Geriatr Soc. 2010;58(6):1063-1071. doi:10.1111/j.1532-5415. 2010.02866.x

7. Soysal P, Veronese N, Thompson T, et al. Relationship between depression and frailty in older adults: a systematic review and meta-analysis. Ageing Res Rev. 2017;36:78-87. doi:10.1016/j.arr.2017.03.005

8. Soderstrom L, Rosenblad A, Thors Adolfsson E, Bergkvist L. Malnutrition is associated with increased mortality in older adults regardless of the cause of death. Br J Nutr. 2017;117(4):532-540. doi:10.1017/ S0007114517000435

9. Kazancioglu R, Buyukaydin B, Cinar A, Soysal P, Gorcin B, Isik AT. Malnutrition in elderly patients with renal failure: importance of predialysis period. Eur Geriatr Med. 2014;5(5):303-306. doi:10.1016/j. eurger.2014.07.004

10. Soysal P, Isik AT. Effects of acetylcholinesterase inhibitors on nutritional status in elderly patients with dementia: a 6-month follow-up study. J Nutr Health Aging. 2016;20(4):398-403. doi:10.1007/s12603015-0603-Z

11. Ates Bulut E, Soysal P, Aydin AE, Dokuzlar O, Kocyigit SE, Isik AT. Vitamin B12 deficiency might be related to sarcopenia in older adults. Exp Gerontol. 2017;95:136-140. doi:10.1016/j.exger.2017.05.017

12. Kinney JM. Nutritional frailty, sarcopenia and falls in the elderly. Curr Opin Clin Nutr Metab Care. 2004;7(1):15-20.

13. Hathaway B, Vaezi A, Egloff AM, Smith L, Wasserman-Wincko T, Johnson JT. Frailty measurements and dysphagia in the outpatient setting. Ann Otol Rhinol Laryngol. 2014;123(9):629-635. doi:10.1177/ 0003489414528669

14. Kaiser MJ, Bauer JM, Ramsch C, et al. Validation of the Mini Nutritional Assessment Short-Form (MNA-SF): a practical tool for identification of nutritional status. J Nutr Health Aging. 2009;13(9):782-788.

15. Kim J, Lee Y, Won CW, Lee KE, Chon D. Nutritional status and frailty in community-dwelling older Korean adults: the Korean Frailty and Aging Cohort Study. J Nutr Health Aging. 2018;22(7):774-778. doi:10.1007/s12603-018-1005-9

16. Jurschik P, Botigue T, Nuin C, Lavedan A. [Association between Mini Nutritional Assessment and the Fried frailty index in older people living in the community]. Med Clin (Barc). 2014;143(5):191-195. doi:10.1016/j.medcli.2013.08.009
17. Chang SF. Frailty is a major related factor for at risk of malnutrition in community-dwelling older adults. J Nurs Scholarsh. 2017;49(1):63-72. doi:10.1111/jnu. 12258

18. Fried LP, Tangen CM, Walston J, et al. Frailty in older adults: evidence for a phenotype. J Gerontol A Biol Sci Med Sci. 2001;56(3):M146-M156.

19. Gungen C, Ertan T, Eker E, Yasar R, Engin F. [Reliability and validity of the standardized Mini Mental State Examination in the diagnosis of mild dementia in Turkish population]. Turk Psikiyatri Derg. 2002; 13(4):273-281.

20. Durmaz B, Soysal P, Ellidokuz H, Isik AT. Validity and reliability of geriatric depression scale-15 (short form) in Turkish older adults. North Clin Istanb. 2018;5(3):216-220. doi:10.14744/nci.2017.85047

21. Lawton MP, Brody EM. Assessment of older people: self-maintaining and instrumental activities of daily living. Gerontologist. 1969;9(3): 179-186.

22. Tinetti ME. Performance-oriented assessment of mobility problems in elderly patients. J Am Geriatr Soc. 1986;34(2):119-126.

23. Vellas B, Guigoz Y, Garry PJ, et al. The Mini Nutritional Assessment (MNA) and its use in grading the nutritional state of elderly patients. Nutrition. 1999;15(2):116-122.

24. Uchmanowicz I, Jankowska-Polanska B, Loboz-Rudnicka M, Manulik S, Loboz-Grudzien K, Gobbens RJ. Cross-cultural adaptation and reliability testing of the Tilburg Frailty Indicator for optimizing care of Polish patients with frailty syndrome. Clin Interv Aging. 2014; 9:997-1001. doi:10.2147/CIA.S64853

25. Alencar MA, Dias JM, Figueiredo LC, Dias RC. Handgrip strength in elderly with dementia: study of reliability. Rev Bras Fisioter. 2012; 16(6):510-514.

26. Sarikaya D, Halil M, Kuyumcu ME, et al. Mini nutritional assessment test long and short form are valid screening tools in Turkish older adults. Arch Gerontol Geriatr. 2015;61(1):56-60. doi:10.1016/j.archger. 2015.04.006

27. Cesari M, Leeuwenburgh C, Lauretani F, et al. Frailty syndrome and skeletal muscle: results from the Invecchiare in Chianti study. Am J Clin Nutr. 2006;83(5):1142-1148. doi:10.1093/ajcn/83.5.1142

28. Soysal P, Stubbs B, Lucato P, et al. Inflammation and frailty in the elderly: a systematic review and meta-analysis. Ageing Res Rev. 2016; 31:1-8. doi:10.1016/j.arr.2016.08.006

29. Soysal P, Isik AT, Arik F, Kalan U, Eyvaz A, Veronese N. Validity of the mini-nutritional assessment scale for evaluating frailty status in older adults. J Am Med Dir Assoc. 2019;20(2):183-187. doi:10.1016/j. jamda.2018.07.016

30. Dokuzlar O, Soysal P, Isik AT. Association between serum vitamin B12 level and frailty in older adults. North Clin Istanb. 2017;4(1):22-28. doi:10.14744/nci.2017.82787

31. Lilamand M, Kelaiditi E, Cesari M, et al. Validation of the mini nutritional assessment-short form in a population of frail elders without disability. Analysis of the toulouse frailty platform population in 2013. J Nutr Health Aging. 2015;19(5):570-574. doi:10.1007/s12603015-0457-4

32. Chang SF, Lin HC, Cheng CL. The relationship of frailty and hospitalization among older people: evidence from a meta-analysis. $J$ Nurs Scholarsh. 2018;50(4):383-391. doi:10.1111/jnu.12397

33. Ruan Q, Yu Z, Chen M, Bao Z, Li J, He W. Cognitive frailty, a novel target for the prevention of elderly dependency. Ageing Res Rev. 2015; 20:1-10. doi:10.1016/j.arr.2014.12.004

34. Fitten LJ. Psychological frailty in the aging patient. Nestle Nutr Inst Workshop Ser. 2015;83:45-53. doi:10.1159/000382060

35. Eeles E, Low Choy N. Frailty and mobility. Interdiscip Top Gerontol Geriatr. 2015;41:107-120. doi:10.1159/000381200 


\section{Publish your work in this journal}

Clinical Interventions in Aging is an international, peer-reviewed journal focusing on evidence-based reports on the value or lack thereof of treatments intended to prevent or delay the onset of maladaptive correlates of aging in human beings. This journal is indexed on PubMed Central, MedLine,
CAS, Scopus and the Elsevier Bibliographic databases. The manuscript management system is completely online and includes a very quick and fair peer-review system, which is all easy to use. Visit http://www.dovepress. $\mathrm{com} /$ testimonials.php to read real quotes from published authors. 